\title{
GESTIÓN DE LA IMAGEN PRESIDENCIAL DEL PERU A TRAVÉS DE UN SISTEMA DE INFORMACIÓN GERENCIAL*
}

Prieto Campos, Carlos ${ }^{1}$ y Sucasaire Portillo, Luz ${ }^{2}$

Facultad de Ciencias de la Comunicación de la Universidad Nacional del Centro del Perú

\begin{abstract}
RESUMEN
El objetivo de la investigación ha sido crear un Sistema de Información Gerencial que mejore la imagen de la Presidencia de la República del Perú. Para ello, fue preciso elaborar un software que permitiera procesar información que conduzca a tomar decisiones para mejorar la imagen presidencial. La información recolectada de los medios de comunicación seleccionados nos permitió ingresar al software información relacionada al Presidente, durante tres meses del año 2008. En una segunda etapa la información ingresada fue procesada, arrojando resultados tanto positivos como negativos acerca de la imagen presidencial. Pero, en este experimento los resultados fueron favorables para el Presidente de la República del Perú. De esta manera, la información procesada por el SIG, de los diarios Perú 21 y La República se ha convertido en información de inteligencia que puede evitar que la imagen del Presidente de la República se desgaste desde el punto de vista político ante la opinión pública.
\end{abstract}

Palabras clave: imagen, SIG, imagen presidencial

\section{MANAGEMENT OF THE IMAGE OF THE PRESIDENCE OF REPUBLICA OF PERU.}

\begin{abstract}
The objective of this research has been to create an information management system to improve the Image of the Republic Presidence of Peru. For this it was necessary to make a software that permits us process information which leads to take decision to improve presidential image. The gotten information from selected communicators let us to get in information into the software, related to the president, during three months in 2008. In the second stage the gotten information was processed, giving both positive and negative results about the presidential image. However, in this experiment the results were helpful to the president of Peru. Thus, the processed information by the SIG from the newspapers Peru 21 and La Republica have become into information of intelligence which can avoid our president's image gets down from a political point of view toward the public opinion.
\end{abstract}

Palabras Clave: image, SIG, presidential image

\footnotetext{
* Este trabajo de investigación fue recibido el 20/03/2009 retornado para su revisión 26/10/2009 y aprobado para su publicación 16/11/2009

'Email: capi06@yahoo.com

2Email: sucaport @yahoo.com
} 


\section{INTRODUCCIÓN}

La Presidencia de la República del Perú, es el órgano ejecutivo del más alto rango en el Perú. El problema de imagen de los jefes de estado cobra cada día mayor importancia en las democracias de todo el mundo debido a la constante vigilancia del ejercicio de su poder por parte de los medios de comunicación, lo que deriva en severas críticas cuando comete yerros. La imagen de la Presidencia de la República en nuestro país, se deteriora en la medida que se incrementan las críticas en forma directa contra su persona o por su entorno en el que está rodeado. Los instrumentos con los que se cuestiona severamente a la Presidencia de la República son principalmente los medios de comunicación: la televisión, la radio, la prensa escrita e internet.

Estas críticas o comentarios negativos que se dan a través de los medios de comunicación pueden ser sistematizados mecánicamente o con tecnología audiovisual en la televisión y hemerográfica en la prensa escrita para realizar el análisis político y estratégico. De esta manera, se puede contrarrestar la imagen negativa que se presenta del Presidente de la República. Sin embargo, estos mecanismos de análisis aún no han sido integrados en su totalidad; para lograr un análisis político y técnico en el tiempo que provoque una respuesta rápida y adecuada para "subsanar" o contrarrestar la imagen negativa contra el primer mandatario, en los medios.

Nuestra hipótesis general fue que el Sistema de Información Gerencial mejora la imagen de la Presidencia de la República del Perú, la cual fue demostrada con el experimento realizado durante tres meses, luego de haber creado el software para tal finalidad.

\section{MATERIALES Y MÉTODOS}

\section{METODOLOGíA.}

\section{Tipo de investigación}

El tipo de investigación de acuerdo a la naturaleza y nivel de la investigación fue básica pura de carácter descriptivo - explicativa, tomando en cuenta las variables utilizadas en la hipótesis planteada.

\section{Diseño de la investigación}

El diseño que utilizamos para la creación del SIG fue experimental

\section{Objeto de estudio}

La Presidencia de la República del Perú y su entorno que lo rodea.

\section{Unidades de análisis}

Informaciones, noticias, reportajes, discurso, comentarios.

\section{Población de estudio}

Los medios de comunicación que tienen una distribución 0 alcance nacional en todo el país. Para este trabajo de investigación se consideran a los medios de comunicación que tienen alcance nacional y son editados desde la ciudad de Lima y que suman 40 .

\section{Cálculo del tamaño de la muestra}

Se consideró un muestreo estratificado con afijación proporcional para cada estrato (radio, TV y medios escritos), luego de determinar el tamaño de la muestra se determinó el número de elementos muéstrales para cada estrato en forma proporcional. En este caso se hizo en forma aleatoria.

La muestra tomada ha sido de los medios de comunicación que se editan, imprimen y distribuyen desde la ciudad de Lima y que tienen una circulación 0 alcance nacional.

$$
\begin{aligned}
& \mathrm{n}=\frac{\mathrm{N} \times \mathrm{Z}_{\mathrm{a}}^{2} \times \mathrm{p}(1-\mathrm{p})}{\mathrm{d}^{2} \times(\mathrm{N}-1)+\mathrm{Z}_{\mathrm{a}}^{2} \times \mathrm{p}(1-\mathrm{p})} \\
& \mathrm{Donde:} \\
& \mathrm{N}=\text { Total de la población }
\end{aligned}
$$

$$
Z_{\alpha}^{2}=1.962^{2}
$$

(Si el nivel de confianza o seguridad es del 95\%)

$p=$ proporción esperada (en este caso $10 \%=0.10$ )

$d=$ precisión (en este caso deseamos un $5 \%$ ).

Aplicando la fórmula tendremos:

Para este caso la muestra fue de los periódicos:

Diario La República

Diario Perú 21 


\section{Técnica del recojo de datos.}

La técnica de recojo de la información para el marco teórico se efectuó a través de la información de bibliografía especializada del análisis de los datos. La técnica de recojo de la información que demostró nuestra hipótesis de funcionamiento del SIG, y la demostración de cómo el SIG gestiona información que ayuda a mejorar la imagen presidencial se realizó una vez concluida la construcción del mismo.

\section{Procedimiento y técnicas:}

Para la recopilación de la información básica del estudio de la investigación hicimos uso de instrumentos confiables

Como:

- Entrevistas a periodistas, ejecutivos, ingenieros de sistemas.

- Grabaciones de audio de noticiarios

- Grabaciones de video de noticiarios

- Encuestas escritas a periodistas y expertos en análisis político

- Observación

- Fichaje bibliográfico.

- Recorte de periódicos señalados

Para el análisis de los datos recopilados se trabajó con lo siguiente:

- Cuantificación

- Cualificación

- Tabulación

- Interpretación de datos

- Tablas

- Análisis de significados

\section{Resultado.}

- Un Sistema de Información Gerencial creado.

- El sistema procesa, analiza información y arroja resultados

- El sistema contribuye a mejorar la imagen del Presidente del Perú

\section{DISCUSIÓN}

\section{Sobre el SIG}

El SIG, Sistema de Información de Gestión de la imagen presidencial, nos muestra en su primera versión su efectividad en el procesamiento de la data de los periódicos seleccionados para nuestra muestra presentada del 22 de febrero al 31 de marzo del 2008. La estructura interna del sistema, está diseñada con un soporte que permite introducir un diccionario de términos relacionados con la imagen positiva o negativa del Presidente de la República. Este elemento diccionario es utilizado para determinar dos categorías para procesar la información seleccionada de los diarios Perú 21 y La República durante los 39 días indicados.

Es cierto que nos muestra una efectividad en el procesamiento de la información, pero limitada, en la medida que hemos considerado sólo un conjunto de términos para el resultado del experimento que mejorará el sistema en una segunda versión; al clasificarse más términos especializados.

Las categorías utilizadas para el trabajo de investigación fueron dos: imagen positiva e imagen negativa del Presidente de la República Dr. Alan García Pérez. El sistema efectúa un trabajo de importación de datos inicialmente. Es decir, importa cualquier archivo o documento. Para nuestro caso, toda la información seleccionada del 22 de febrero al 31 de marzo del 2008 de los diarios Perú 21 y La República.

Una vez importada la información de los periódicos, definimos nuestras unidades de análisis a través de las noticias, editoriales y comentarios de opinión que se divulgaban en dichos medios indicados e inmediatamente iniciábamos el procesamiento de dichos datos en el SIG, para observar su comprobación y aplicabilidad, reconociendo que el sistema sí, procesa información para proyectar las tendencias de la imagen positiva y negativa del Presidente de la República. En este caso, la proyección de las noticias, editoriales y comentarios de opinión que mencionaban o estaban relacionados con el Primer Mandatario de la Nación.

El Sistema arrojó dos resultados: la imagen positiva y la imagen negativa del Jefe del Estado, sin embargo, 
éstos resultados son aproximaciones valiosas, pero no; determinantes en un 100\%; debido a que nuestra primera versión del software nos ayuda a observar cómo va la tendencia con una confiabilidad de $60 \%$; afirmando que el sistema contribuye a conocer la imagen presidencial en sus dos categorías negativa y positiva.

Toda la información procesada, palabras relacionadas con el presidente, en su primera fase fue traslada inmediatamente al programa Excel, donde finalmente se comprobó cuantitativamente y porcentualmente como aparece la imagen presidencial, tomando como referencia la información mediatizada de las muestras estudiadas y analizadas por día y de manera general.

Finalmente, obtener la información procesada del SIG y presentarla adecuadamente en figuras y barras estadísticas facilita la interpretación sobre la imagen presidencial para el análisis del trabajo en equipo.

\section{Sobre la toma de decisión para mejorar la imagen presidencial}

En los gráficos generales resumidos presentados: Gráfico $N^{0} 39$ y 40, la tendencia de la imagen presidencial del 22 de febrero al 31 de marzo del 2008 es positiva de acuerdo con las unidades de análisis estudiadas y analizadas que nos proporcionó el software del sistema realizado.

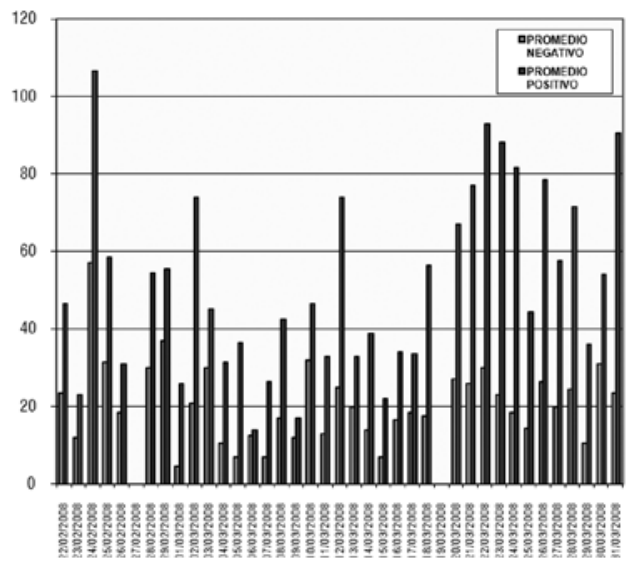

Gráfico 1. Resumen de imagen presidencial positivo - negativo.

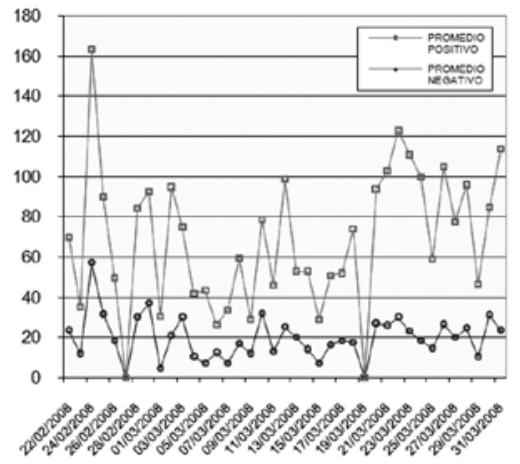

Gráfico 2. Resumen de imagen presidencial promedio positivo

\section{CONCLUSIONES}

- El SIG, Sistema de Información de Gestión de la imagen del Presidente Dr. Alan García Pérez, en esta primera versión del software realizado, logra procesar y analizar información de los medios de comunicación para tomar decisiones que mejoren la imagen presidencial.

- El obtener información clasificada de los medios de comunicación sobre el Presidente, permite que Ios analistas realicen un mejor trabajo, en primer lugar por la rapidez en la que se obtiene la información y porque se puede anticipar o planear nuevos eventos para las próximas publicaciones de los medios, en la medida que se estudia las proyecciones que los periodistas harán en sus comentarios futuros.

- De acuerdo con los resultados que nos proporciona el SIG, de la muestra tomada de los diarios seleccionados Perú 21 y La República, desde el día 22 de febrero al 31 de marzo, podemos concluir que la imagen presidencial tiene una tendencia positiva.

- Podemos deducir del experimento, que la imagen positiva que se proyecta en el diario La República proviene de las noticias y comentarios de opinión generados por los periodistas en las que se destacan las actividades positivas.

- La primera decisión a tomar de acuerdo con los re- 
sultados obtenidos del SIG, en nuestro experimento con el Presidente Alan García, debe de ser por ejemplo, que sus actividades 0 acciones positivas divulgadas como noticias u opinión en los medios de comunicación Perú 21 y La República, sean replicadas poniéndolas de relieve en todos los medios de comunicación.

- Concluimos también que los discursos siempre deben ser enfocados con términos positivos. Es decir, el contenido de la temática debe considerar palabras, frases y oraciones, verbos, sustantivos y adjetivos cuyo contenido sea positivo.

- El diario La República de acuerdo al análisis e interpretación realizados de los datos obtenidos por el SIG, es el diario de mayor temática, noticias, editoriales, comentarios y otros aborda sobre el Presidente de la República Dr. Alan García Pérez. Nos muestran que las informaciones tienen mayormente un carácter positivo que negativo.

- Las noticias y los comentarios de opinión son la materia prima para que exista la opinión pública. Y si las noticias en su mayoría tienen temática positiva, la opinión pública va a ser positiva o buena, porque el clima es positivo y por lo tanto las tendencias observadas en el análisis que realiza el SIG, son idénticas o positivas.

- El diario Perú 21, divulga poca información temática relacionada con el Presidente de la República mostrando por el contrario, mayor información de carácter negativa de acuerdo con la data proporcionada y procesada por el SIG. La información negativa que se difunde en Perú 21, genera opinión negativa. El colectivo de la ciudadanía percibe a través de este medio que las cosas no están bien y que el Presidente de la República se encuentra gestionando un mal gobierno.

- EI SIG, ayuda a contrarrestar aquella opinión pública negativa, si el sistema recoge y procesa la mayor cantidad de información negativa publicada en los medios, para luego analizarla y después de breves minutos planear y ejecutar eventos o escenarios favorables. Estos, deberán generar noticias positivas en los medios de comunicación; cuyo efecto será la mejora de la imagen presidencial.
- De acuerdo con el resultado general, en el gráfico No 39 y 40, podemos concluir que la tendencia de la imagen presidencial del 22 de febrero al 31 de marzo del 2008, es positiva de conformidad con las unidades de análisis estudiadas.

- El sistema al generalizar y representar la data analizada durante un período de tiempo permite gestionar la imagen presidencial con eficiencia y eficacia. De ésta forma, se va llevando un historial en archivo, que va a servir para los meses y años venideros. Además, el trabajo del equipo de análisis será más creativo por las comparaciones que se pueden realizar en un par de gráficos.

- El SIG creado, contribuye a tomar decisiones para mejorar o mantener la imagen del Presidente de la República Dr. Alan García Pérez, luego de conocer el resultado del procesamiento y análisis e interpretación realizado a los diarios Perú 21 y La República del 22 de febrero al 31 de marzo del 2008.

- La información procesada por el SIG, de los Diarios Perú 21 y La República se ha convertido en información de inteligencia para los intereses del gobierno al evitar que la imagen del Presidente de la República se desgaste desde el punto de vista político ante la opinión pública. Con dicha información de inteligencia se fortalecerán los planes y estrategias de comunicación para generar una buena opinión pública para el Primer Mandatario de la Nación y por ende al gobierno democrático.

\section{LECTURA CITADA}

Abram A. Moles. 2000. Teoría estructural de la comunicación y sociedad. Editorial Trillas. México D.F.

Druker, Peter. 1996. La administración basada en la información. Grupo Editorial Norma S.A. Bogotá. Colombia.

Henri Pierre. 1995 La Casa Blanca. En los años de Reagan y Bush. Ediciones Javier Vergara, Argentina.

Miguel Santemases Mestre. 2001. Marketing. Conceptos y estrategias. Ediciones Pirámide. Madrid. 
Myer G. David. 1997. Psicología social. Editorial Mc Graw Hill. Ediciones. México.

Noelle - Neumann Elizabeth. 1997. La espiral del silencio. Ediciones Paidós. España.

Scheinsonh Daniel A. 1996. Comunicación estratégica. Editorial Norma. Colombia.

Van Riel Gees B.M. 2000. Comunicación corporativa. Ediciones Prentice Hall. España.
Páginas electrónicas:

http://www.gestiopolis.com/canales/gerencial/articulos/59/pronosfut.htm

http://www.monografias.com/trabajos17/sistemagerencial/sistema-gerencial.shtml

http://ww.cinterfor.org.uy/public/spanish/region/ampro/cinterfor/temas/youth/exp/bol/procal/sgi.htm

http://es.wikipedia.org/wiki/Sistemas_de_ informaci\%C3\%B3n_gerencial 